\title{
Left-right symmetry breaking in mice by left-right dynein may occur via a biased chromatid segregation mechanism, without directly involving the Nodal gene
}

\author{
Stephan Sauer* and Amar J. S. Klar* \\ Gene Regulation and Chromosome Biology Laboratory, Frederick National Laboratory for Cancer Research, Frederick, MD, USA
}

\section{Edited by:}

James L. Sherley, Boston Biomedical Research Institute, USA

\section{Reviewed by:}

Paola Parrella, IRCCS Casa Sollievo Della Sofferenza, Italy

Mitsuru Furusawa, Neo-Morgan Laboratory Incorporated, Japan

*Correspondence:

Stephan Sauer and Amar J. S. Klar, Gene Regulation and Chromosome Biology Laboratory, Frederick National Lab for Cancer Research, 7th Street Fort Detrick, Frederick, MD 21702, USA.

e-mail: sauers@mail.nih.gov; klara@mail.nih.gov
Ever since cloning the classic iv (inversed viscerum) mutation identified the "left-right dynein" (Ird) gene in mice, most research on body laterality determination has focused on its function in motile cilia at the node embryonic organizer. This model is attractive, as it links chirality of cilia architecture to asymmetry development. However, Ird is also expressed in blastocysts and embryonic stem cells, where it was shown to bias the segregation of recombined sister chromatids away from each other in mitosis. These data suggested that $I r d$ is part of a cellular mechanism that recognizes and selectively segregates sister chromatids based on their replication history: old "Watson" versus old "Crick" strands. We previously proposed that the mouse left-right axis is established via an asymmetric cell division prior to/or during gastrulation. In this model, left-right dynein selectively segregates epigenetically differentiated sister chromatids harboring a hypothetical "left-right axis development 1" ("Ira1") gene during the left-right axis establishing cell division. Here, asymmetry development would be ultimately governed by the chirality of the cytoskeleton and the DNA molecule. Our model predicts that randomization of chromatid segregation in Ird mutants should produce embryos with $25 \%$ situs solitus, 25\% situs inversus, and $50 \%$ embryonic death due to heterotaxia and isomerism. Here we confirmed this prediction by using two distinct Ird mutant alleles. Other than Ird, thus far Nodal gene is the most upstream function implicated in visceral organs laterality determination. We next tested whether the Nodal gene constitutes the Ira1 gene hypothesized in the model by testing mutant's effect on $50 \%$ embryonic lethality observed in Ird mutants. Since Nodal mutation did not suppress lethality, we conclude that Nodal is not equivalent to the Ira1 gene. In summary, we describe the origin of $50 \%$ lethality in Ird mutant mice not yet explained by any other laterality-generating hypothesis.

Keywords: laterality development, left-right dynein, asymmetric cell division, DNA strands differentiation, selective chromatid segregation

\section{INTRODUCTION}

It is crucial for multicellular development that cells possess a memory system, which ensures stable inheritance of acquired developmental states during development of tissues and organs of an organism. The field of epigenetics studies this cellular memory system, and "epigenetic" is often defined as "mitotically heritable changes in gene expression that do not involve modulation of the primary DNA sequence." For development, it is equally important that cells are able to change their acquired developmental state and differentiate along evolutionarily defined lineage paths. A crucial question is how epigenetic information can be changed and passed onto developmentally differentiated sister cells during asymmetric cell division. We proposed a solution to this problem. Namely, sister chromatids can be epigenetically differentiated regarding a developmentally important gene during S-Phase, based on lagging versus leading strand DNA replication, followed by selective sister chromatid segregation to specific daughter cells (Figure 1A). Our Somatic Strand-specific Imprinting and selective sister chromatid Segregation (SSIS) model (Klar, 1994) postulates that a specific daughter inherits both template Watson and first time synthesized Crick strand-containing (WC') homologous chromosomes, thereby the other daughter inherits with both new Watson and old Crick $\left(\mathrm{W}^{\prime} \mathrm{C}\right)$ homologous chromosomes (referred to as WW:CC segregation pattern). As a consequence, a single gene or a gene cluster is poised for expression in one daughter cell and silenced in the other daughter cell. Likewise, if sister chromatids were selectively segregated in a WC:WC fashion, then both daughter cells would inherit equivalent epigenetic make ups and hence retain similar developmental potentials, as seen in symmetrical stem cell divisions. The SSIS model is based on studies on fission yeast (Schizosaccharomyces pombe) mating-type switching (Klar, 2007), and has been tested in vitro in mouse embryonic stem (ES) cells (Armakolas and Klar, 2006, 2007), and in vivo in a mouse model for body laterality development (this study).

Schizosaccharomyces pombe is a haploid unicellular eukaryote, whose cells either express $\mathrm{P}$ or M mating-type information from the alternate alleles of the mat1 locus residing in chromosome 2. The mat1 mating-type content switches between $\mathrm{M}$ and P information by a cell cycle controlled DNA transposition 
A

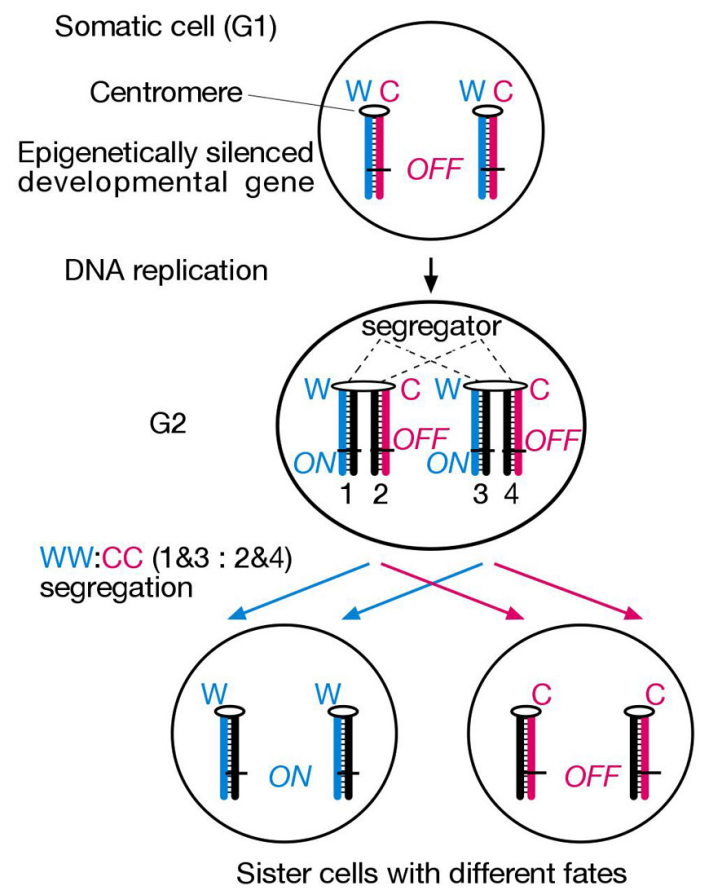

FIGURE 1 | Strand-specific imprinting in diploid and haploid organisms. (A) Hypothetical asymmetric cell division according to our strand-specific imprinting and selective segregation (SSIS) model. Only one pair of homologous chromosomes is illustrated. Lagging versus leading strand DNA replication epigenetically differentiates an important developmental gene on sister chromatids, $O N$ in one and $O F F$ in the other. A segregator, such as left-right dynein, "sorts" sister centomeres/chromatids according to their replication history in G2, causing selective segregation of older Watson template strands into specific daughter cell, and older Crick template strands into the other daughter cell (named WW:CC segregation). Hence, asymmetric DNA replication-coupled epigenetic chromatin modification and selective sister chromatid segregation in the parent cell can specify different developmental potentials to daughter cells (Klar, 1994). Symbols: W, template
B

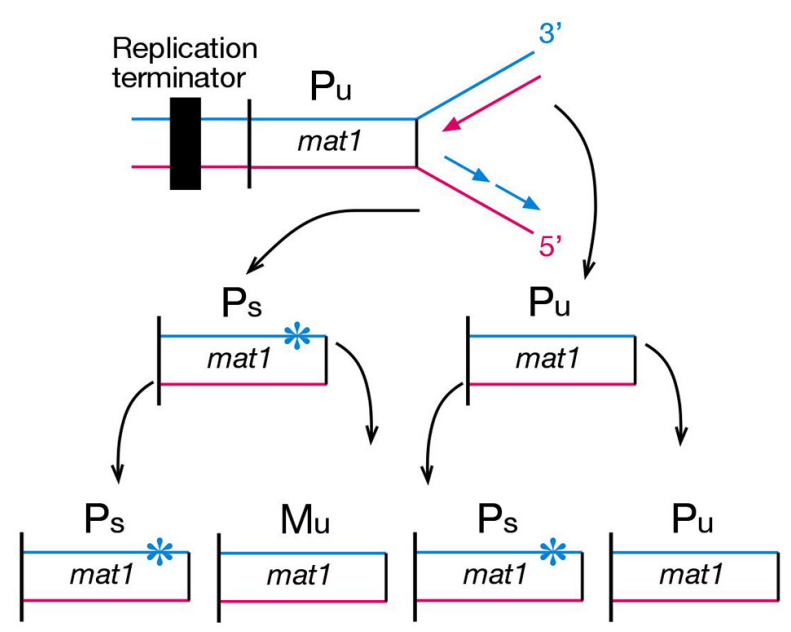

"Watson" strand, C, template "Crick" strand. Numbers 1-4 represent specific chromatids with respect to their strands' constitution. (B) Illustration of how lagging strand-specific imprinting explains the " 1 in 4 granddaughters switching" rule in $S$. pombe mating-type switching. The mat 1 locus efficiently switches $\mathrm{P}$ and $\mathrm{M}$ mating-type gene information by a cell cycle controlled DNA transposition mechanism. A replication terminator ensures unidirectional DNA replication of the mat 1 locus, and lagging strand DNA synthesis installs an imprint (indicated by star) in a sequence- and strand-specific manner in an unswitcable (Pu) cell. The imprint confers competence for switching at the mat1 locus only in the daughter cell inheriting the imprinted chromosome (Ps), which transposes opposite mating-type information copied from the silenced donor loci into the mat1 locus only in one of the sister chromatids (Klar, 2007). mechanism, such that one out of four granddaughter cells switches cell type and expresses the mating-type opposite to that of the grandmother cell (Figure 1B). Genetic and biochemical analysis revealed that mating-type switching is controlled by laggingversus leading-strand DNA replication at the mat1 locus. In particular, lagging-strand DNA synthesis installs an imprint at mat1 (most probably a two nucleotide long DNA:RNA hybrid from an incompletely removed Okazaki fragment), which initiates a double-strand break during the following S-Phase to start the DNA transposition event that underlies mat1 switching. Hence developmental asymmetry between sister cells can be traced back to double helical structure of the mat1 gene and lagging- versus leadingstrand synthesis of specific DNA strands in two consecutive cell divisions (Klar, 2007).

We proposed that a similar mechanism might produce asymmetric cell divisions in diploid organisms by epigenetic means as well. First, strand-specific imprinting would epigenetically differentiate sister chromatids in S-Phase, and selective segregation of thus differentiated sister chromatids would create sister cells with different developmental fates. This model is called SSIS, and was initially developed by us to explain internal organ laterality development in vertebrates (Klar, 1994).

The development of bilateral asymmetry can be conceptually divided into three steps: First comes the initial symmetry-breaking event, usually ascribed to cellular amplification of a molecular chirality. This is followed by differential gene expression in cell fields on either side of the midline, which translates to step three, left/right (L/R) asymmetric organogenesis (Aw and Levin, 2009). For internal organ situs development in vertebrates, a great deal of molecular understanding has been achieved to decipher steps two and three, where many molecular pathways, seemingly conserved between model organisms, have been defined and well accepted (Nakamura and Hamada, 2012). For example, the TGF- $\beta$ related signaling molecule Nodal is conserved in all deuterostomes examined, and usually specifies the left body-side (Chea et al., 2005). Its activity is inhibited toward the midline by Nodal's own transcriptional targets of the Lefty family of diffusible molecules, which represents a prime example of a reaction-diffusion mechanism (Nakamura et al., 2006; Muller et al., 2012). In contrast, identity of the symmetry-breaking event, the "first event," that initiates 
left-biased Nodal expression is controversial, because no unifying mechanism between vertebrate phyla has been in identified to date (Vandenberg and Levin, 2009). Some vertebrates such as mice, frogs, and zebrafish are proposed to employ motile cilia during gastrulation at equivalent embryonic organizer regions, known as the node, gastrocoel roof plate, and Kupffer vesicle, respectively. Cilia's beating is thought to either transport a morphogen leftwards in extraembryonic space (Nonaka et al., 1998), or induce asymmetrical calcium signaling in conjunction with mechanosensory cilia (McGrath et al., 2003). As a consequence, Nodal signaling is induced more strongly in left-sided neighboring tissues (lateral plate mesoderm in the mouse), and its autoregulatory feedback loop with Lefty molecules confers robustness to the signaling cascade (Nakamura and Hamada, 2012). This model is very attractive as it links the molecular chirality of the cilium and its building blocks to chirality of the developing embryo. However, several observations prominently question this model's universality, and some data would rather support a role for nodal cilia during step two of bilateral asymmetry development, namely, asymmetric gene expression on either side of the midline. First, pigs, for example, undergo L/R axis development without motile nodal cilia, undermining a universal role for motile cilia in vertebrate and mammalian symmetry-breaking (Vandenberg and Levin, 2010). Second, in species that employ cilia, a number of genes that are required for proper nodal cilia motility and positioning are also expressed in non-ciliated cells at much earlier embryonic stages. Examples include planar cell polarity genes Vangl2 and Dvl2, inversin and left-right dynein (Aw and Levin, 2009). Thus, it is unclear whether these proteins exert their critical function in L/R axis development at the node. Third, mouse blastomere cells rearrangement has been shown to influence direction of embryonic turning, indicating that some aspects of laterality development certainly occur prior to gastrulation, and are independent of nodal cilia (Gardner, 2010). Last, both zebrafish and mouse mutants have been isolated, which show Kupffer vesicle or node ciliary defects but no L/R phenotypes, and vice versa (Vandenberg and Levin, 2010). Therefore, despite overwhelming evidence suggesting that cilia do have an important function in L/R asymmetry development in several species, they are unlikely to truly control initial symmetry-breaking in the embryo to generate L/R asymmetry (Tabin, 2005; Klar, 2008; Lobikin et al., 2012).

In 1959, Hummel and Chapman (1959) first described the recessive iv (inversed viscerum) mutation, where $50 \%$ of homozygous mice develop situs inversus (i.e., mirror-image reversal of internal organs), and 50\% have normal organ situs. Parental organ situs does not affect organ situs of the offspring, thus this mutation randomizes L/R asymmetry. More detailed analysis revealed high rates of heterotaxia (random and independent sidedness of internal organs) affecting both normal and situs inversus homozygous mutants at similar rates and severity (Layton, 1978). This suggests that in addition to its involvement in the first step of asymmetry development, the iv gene product is also needed in the second and/or third conceptual steps described above. Molecular cloning by Supp et al. (1997) showed that the iv mutation changed a highly conserved glutamic acid to lysine within the motor domain of a dynein heavy chain gene, which was thereafter named left-right dynein (lrd). Lrd message was detected in blastocysts and (blastocyst-derived) ES cells, ventral node cells, and some ciliated embryonic and adult epithelia. It was classified as an axonemal dynein despite its obvious expression in many nonciliated cell types. At the time the authors were not aware that node cells contain motile cilia, and even concluded that: ". . embryonic expression indicates that mechanisms other than ciliary movement are involved in L/R specification" (Supp et al., 1997). Later it was found that Node cells contained ciliated cells (Nonaka et al., 1998) whose motility is dependent on lrd (Supp et al., 1999). Technically difficult studies further showed that beating nodal cilia created a leftward fluid-flow in extraembryonic space (Nonaka et al., 1998), and artificial fluid-flow reversal had a dominant effect on situs development in normal and lrd mutant embryos (Nonaka et al., 2002). These data clearly highlight the node's function as an embryonic organizer during $\mathrm{L} / \mathrm{R}$ axis development. Whether the $\mathrm{L} / \mathrm{R}$ asymmetry is truly established by nodal flow or whether this simply represents a "back-up" mechanism remains to be determined. To address this question, our lab has started to generate a conditional allele for $\mathrm{lrd}$ to discriminate between early cytoplasmic and later axonemal (cilia) functions.

A study from our lab has provided genetic evidence that $1 \mathrm{rd}$ does indeed have a functional role in non-ciliated cells (Armakolas and Klar, 2007). Liu et al. (2002) had engineered mouse ES cells lines, which allowed for selection of Cre/loxP-mediated mitotic recombinants between homologs. If recombination happens in G2, recombined chromatids can either segregate together ( $Z$ segregation) or into different sister cells ( $\mathrm{X}$ segregation). $\mathrm{X}$ segregants thereby acquire homozygosity of any heterozygous marker distal to the crossover site. Interestingly, centromere-proximal loxP sites on chromosome 7 (DT1E9 cell line) always led to X segregation, whereas loxP sites on chromosome 11 or further centromeredistal on chromosome 7 , produced the usually expected random mix of $X$ and $Z$ segregants (Liu et al., 2002). Differentiation of DT1E9 ES cells to endoderm cells preserved the exclusive X segregation pattern, whereas neuroectoderm cells showed exclusive $Z$ segregation. Three other in vitro differentiated cell types showed random patterns (Armakolas and Klar, 2006). We proposed that cell type-specific biased segregation patterns were due to selective chromatid recombination as well as selective segregation of chromosome 7 sister chromatids in mitosis. Remarkably, lrd mRNA expression was evident in ES, endoderm, and neuroectoderm cells, and RNAi-mediated knockdown randomized segregation patterns, consistent with our SSIS model (Klar, 2008). In this model, lrd would "sort" sister chromatids based on their replication history and selectively segregate sister centromeres to sister cells (Figure 1A). We propose that $l r d$ 's function in non-ciliated cells is to bias sister chromatid segregation of one or a specific set of chromosomes. By theory, this function is not confined to a single $\mathrm{L} / \mathrm{R}$ axis establishing asymmetric cell division, but probably happens in other developmental contexts where asymmetric or strictly symmetric cell divisions occur. Additional support for this is provided by $l r d$ 's expression profile available on the gene annotation portal biogps.org, where lrd shows high expression in hematopoietic stem cells (http://www.biogps.org/\#goto). Here we tested developmental biology predictions of the SSIS model concerning the lrd mutant. In a second experiment we tested whether 
the Nodal gene comprises the "left-right axis development 1" (lra1) gene specified in the SSIS model.

\section{MATERIALS AND METHODS \\ MOUSE BREEDING AND HUSBANDRY}

Lrd-Neo-GFP mice were a kind gift from Dr. Martina Brueckner at Yale University, New Haven, CT. The iv stock (EM:02531) was purchased (live) from EMMA repository, Harwell, UK. Delta Nodal mice were a kind gift from Dr. Michael Kuehn, Frederick National Laboratory, MD. All mice were kept according to Animal Care and User Committee (ACUC) guidelines, Frederick National Laboratory, MD.

\section{GENOTYPING}

Between 3 and 4 weeks of age, tailclips were performed according to ACUC guidelines. Tails were digested by overnight incubation at $55^{\circ} \mathrm{C}$ in $200 \mu \mathrm{l}$ of tail buffer [ $100 \mathrm{mM} \mathrm{NaCl}, 10 \mathrm{mM}$ Tris- $\mathrm{HCl}$ $\mathrm{pH} 7.5,10 \mathrm{mM}$ EDTA, 0.5\% (w/v) $N$-Lauroylsarcosine, $100 \mu \mathrm{g} / \mathrm{ml}$ Proteinase K]. The solution was then diluted 1:1 with $\mathrm{dH}_{2} \mathrm{O}, 1 \mu \mathrm{l}$ was used for PCR reactions. Lrd-Neo-GFP primers: wtaF3: CTCTGCAGGCAGAGCGGCT, taR3: GCTTGCCGGTGGTGCAGA, wtR3: CGGGTCTAGGGCAAAGCGTT. PCR: $95^{\circ} \mathrm{C} 2 \mathrm{~min}-34 \times$ $\left(94^{\circ} \mathrm{C} 20 \mathrm{~s},-64.5^{\circ} \mathrm{C} 20 \mathrm{~s},-72^{\circ} \mathrm{C} 30 \mathrm{~s}\right) 72^{\circ} \mathrm{C} 5 \mathrm{~min}$. wt allele: $194 \mathrm{bp}$, targeted allele: 266 bp. Nodal Delta primers: F4299: CAGAAGAGGGATTTGGGGTTTGCAG, R4457: GATCGGAACTCAGGAACCTAGAAAC. $95^{\circ} \mathrm{C} 2 \mathrm{~min}-32 \times\left(94^{\circ} \mathrm{C} 30 \mathrm{~s},-65^{\circ} \mathrm{C} 30 \mathrm{~s},-72^{\circ} \mathrm{C}\right.$ $30 \mathrm{~s}) 72^{\circ} \mathrm{C} 5 \mathrm{~min}$. Targeted (delta) allele: $\sim 180$ bp. iv primers: 1959 TaqaI F: GCTAACCACCAACCACATGCTG, 1959 TaqaI R: CACGGATTCCAGCCCAGATC. $25 \mu \mathrm{l}$ PCR product was digested with $25 \mathrm{U}$ of Taq alpha $\mathrm{I}(\mathrm{NEB})$ in a $40 \mu \mathrm{l}$ reaction, at $65^{\circ} \mathrm{C}$ for $45 \mathrm{~min}$. The iv mutation destroys the Taq alpha I site in the PCR fragment. wt bands: $92 \mathrm{bp}$, iv band: $184 \mathrm{bp}$.

\section{RESULTS}

\section{A TEST OF A KEY PREDICTION OF THE SSIS MODEL}

Our model makes several testable predictions for the phenotype of the lrd mouse mutant. First, randomization of sister chromatid segregation during the critical L/R axis establishing cell division should have three different outcomes: 25\% WW:CC cell pairs leading to normal organ situs later in development, 25\% CC:WW cell pairs leading to inversed organ situs, and 50\% WC:WC cell pairs causing embryonic lethality or death soon after birth due to isomerism (mirror-image sidedness of organs) or heterotaxia (random and independent sidedness of organs; Figure 2). Lethality occurs because of the lral gene's ON/OFF epiallele constitution in both sister cells. Prediction of 50\% lethality in $l r d$ mutant mice is a major difference between SSIS hypothesis and mainstream nodal cilia hypotheses for $\mathrm{L} / \mathrm{R}$ axis development (Klar, 2008).

We acquired two different $l r d$ mutant mouse strains, the original iv strain from EMMA repository and the Lrd-Neo-GFP mouse from Dr. Martina Brueckner's laboratory (McGrath et al., 2003). The iv strain originated from a complex mixed background until siblings were inbred for $>20$ generations (EMMA repository, personal communication). The Lrd-Neo-GFP allele was introduced into ES cells of Sv129 genetic background (McGrath etal., 2003). To reduce background specific influences, both

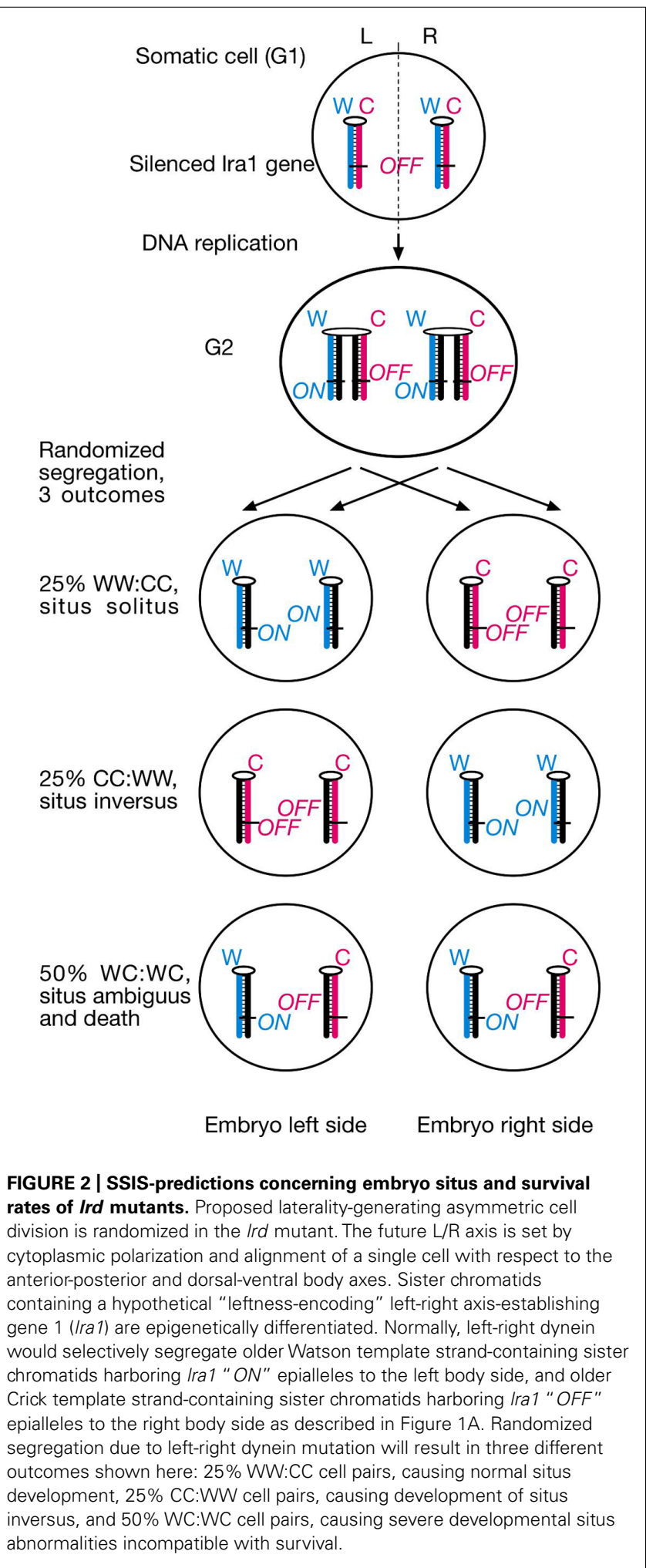

strains were bred onto C57BL/6 strain for one generation. Lethality rates were determined at weaning age (3-4 weeks) by PCR based genotyping of tailclip DNA (see section Materials and Methods). 
Table 1 | Observed rates of allele frequencies: Lrd-Neo-GFP allele, Ird $^{+/-} \times$Ird $^{+/-}$.

\begin{tabular}{lll}
\hline $\mathbf{L r d}^{+/+}(\%)$ & $\boldsymbol{I r d}^{+/-}(\mathbf{\%})$ & $\boldsymbol{I r d}^{-/-}(\%)$ \\
\hline $53(32)$ & $90(55)$ & $22(13)$ \\
\hline
\end{tabular}

Table 2 | Observed rates of allele frequencies: iv allele, $i v^{+/-} \times i v^{-1-}$.

\begin{tabular}{ll}
\hline$i v^{+/-}(\%)$ & $i v^{-1-}(\%)$ \\
\hline $74(67)$ & $37(33)$
\end{tabular}

Lrd-Neo-GFP mice carry a GFP-lrd exon 1 fusion as well as a Neo cassette on the opposite strand of lrd intron 1. Since the Neo transgene is under the control of a very strong promoter and transcribed antisense to $l r d$, lrd transcription is effectively shut down and homozygous mutant mice are indistinguishable from true knockout mice: $50 \%$ of live animals exhibit situs inversus (McGrath et al., 2003). Several heterozygous intercrosses ( $\left.l \mathrm{rd}^{+/-}\right)$were set up and DNA from tails from 165 offspring was analyzed (Table 1). We detected $53 \mathrm{lrd}^{+/+}: 90 \mathrm{lrd}^{+/-}: 22 \mathrm{lrd}^{-/-}$animals. The SSIS hypothesis predicts $\sim 24(165 / 7)$ of live-born mice to be $l r d^{-/-}$. This is because $1 / 8$ (half of $1 / 4$ animals with $-/-$ genotype) of the initial number of homozygous mutant mice is expected to live, $1 / 8$ is expected to die and thus reduce the total number of mice that are available for analysis to $7 / 8$. As a result, $1 / 8$ of the initial mice correspond to $1 / 7$ of observable mice. If lethality was not an issue, then $\sim 41$ mice (1/4 of 165 ) should have the $l \mathrm{rd}^{-/-}$genotype. Our observed number of $22 \mathrm{lrd}-/-$ mice is statistically equivalent to the SSIS-predicted number of 23.57 ( $p$-value of $\sim 0.6$, chisquare test).

Encouraged by the heterozygous cross results, we set up four $i v^{+/-} \mathrm{X} i v^{-/-}$crosses. The results are summarized in Table 2. Conventionally $1 / 2$ of the offspring is expected to be $i v^{-/-}$. However if lethality affected $50 \%$ of the $i v^{-/}$mice, this fraction would be reduced to $1 / 3$ among live animals. Analysis of 111 offspring revealed $74 i v^{+/-}$and $37 i v^{-/-}$mice, which meets SSIS prediction exactly.

\section{DOES NODAL CONSTITUTE THE LRA1 GENE HYPOTHESIZED IN THE SSIS MODEL?}

According to the SSIS model, heterozygosity for the lral gene would prevent embryonic lethality in $i v^{-/}$embryos because heterotaxia or isomerism would not occur. Consequently, 50\% would develop normal organ situs and 50\% would develop situs inversus in embryos with $l \mathrm{ral}^{+/-}, \mathrm{iv}^{-/-}$genotype (Figure 3A). We chose a candidate gene approach, and considered the Nodal gene as a likely candidate for the lral gene as it is the gene, other than $i v$, that functions most upstream in the L/R pathway. Nodal belongs to the TGF- $\beta$ family of extracellular signaling molecules and has been shown to be amongst the earliest asymmetrically (left-sided) expressed molecules in a variety of species, ranging from snails to man (Nakamura and Hamada, 2012). Since Nodal is essential for mesoderm induction during gastrulation and the

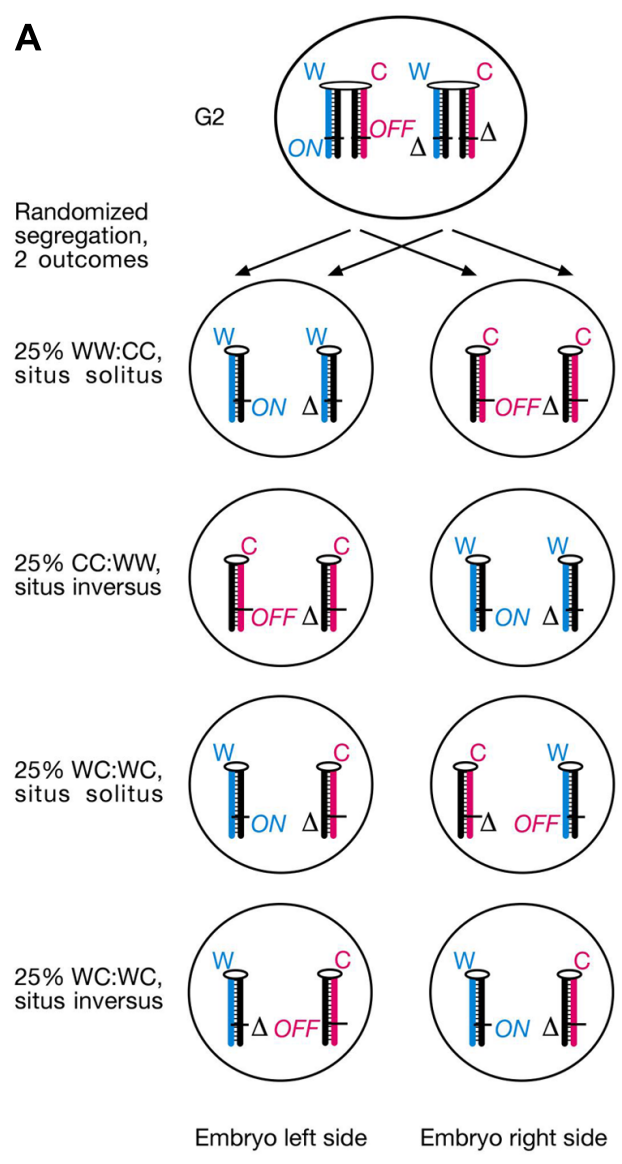

B

iv+l- $\quad$ iv-/-

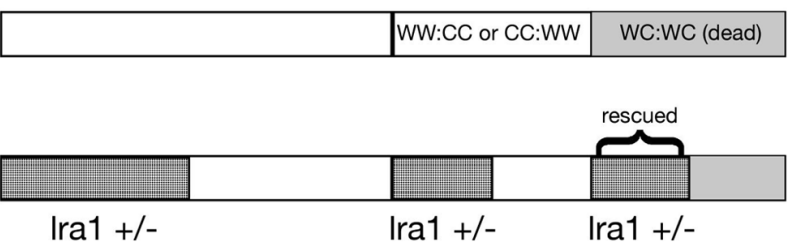

FIGURE 3 | (A) Lra1 heterozygosity is predicted to rescue WC:WC segregants that occur in Ird mutants. As illustrated in Figure 2, SSIS predicts $50 \%$ lethality in Ird mutants due to occurrence of WC:WC segregation at $50 \%$ incidence. Lethality is due to conflicting (ON and OFF) Ira1 epialleles in cells that inherited both older Watson and older Crick template strands. However, in compound Ird homozygous and Ira1 heterozygous mutant embryos, WC:WC segregants are predicted to survive. This is because Ira 1 has only one functional allele, the lethality-causing ON/OFF combination in both sister cells described in Figure 2 cannot be generated. Therefore, a 50:50 distribution of situs solitus and situs inversus animals is expected to develop. Symbols: $\Delta$, deletion of Ira1 ( = Nodal?); rest of symbols are as described in Figure 1A. (B) SSIS-predicted ratios of genotypes from an $i^{+} /-X i v^{-/-}$cross (top) and an iv $+/-$, Ira $1^{+/-X} \mathrm{iv}-/-$, Ira $1^{+/+}$cross (bottom). Conventionally $50 \%$ offspring is expected to be Ira ${ }^{+1-}$. Because WC:WC segregants (gray) are predicted not to die if they are also Ira $1^{+/-}$, Ira $1^{+/-}$animals should be overrepresented in the offspring by a 4:3 ratio. Moreover, iv $v^{-1-}$ are predicted to occur at a 3:4 ratio as opposed to $1: 2$ (top), and $/ \mathrm{ra} 1^{+/-}$ $i v^{-/-}$animals are also predicted to occur at increased rates (2/7). 
mutant embryos die before $\mathrm{L} / \mathrm{R}$ axis is established (Lowe et al., 2001), its function for $L / R$ axis development is difficult to address by using a conventional null allele. Conditional inactivation of the FoxH1 transcriptional activator in the lateral plate mesoderm causes loss of Nodal expression and R/R isomerism (Yamamoto et al., 2003). Likewise, injection of $\mathrm{Nodal}^{-/-}$ES cells into wildtype (wt) blastocysts results in development of R/R isomers ( $\mathrm{Oh}$ and Li, 2002). Thus, Nodal is considered to encode "leftness." If Nodal is in fact the lral gene, the $50 \%$ lethality of $1 r d$ homozygous mutant embryos should be suppressed in $\mathrm{Nodal}^{+/-}$heterozygotes according to our model (Figure 3A).

We determined whether heterozygosity for a null allele of the Nodal gene (Lowe et al., 2001) in iv crosses affected lethality ratios. Specifically, we determined whether the mutation suppresses 50\% lethality of $i v^{-/-}$mice described in Figure 2. Because the Nodal gene deletion is homozygous lethal, we therefore quantitated viability of only heterozygous animals. We generated several males heterozygous for iv and delta Nodal mutations, which we set up with $i^{-/-}, \mathrm{Nodal}^{+/+}$females. Therefore, half of all offspring will be heterozygous for delta Nodal allele. In this mating set up, several predictions concerning ratios of expected genotypes are made (Figure 3B). Should heterozygous Nodal mutation not influence lethality ratios, then $1 / 3 \mathrm{iv}^{-/-}$mice should be observed, just like the result of the cross described in Table 2. Accordingly, 50\% will be heterozygous for the delta Nodal mutation, and 1/6 (1/3 × 1/2) will be both $i v^{-/-}$and carriers of delta Nodal. If heterozygosity for Nodal rescues lethality in WC:WC segregants, then only $1 / 8$ $(1 / 2 \times 1 / 2 \times 1 / 2)$ of initial conceptuses will die, which reduces the total number of observable mice to $7 / 8$. As discussed above, $1 / 8$ of initial mice correspond to $1 / 7$ of observable (live) mice. Three out of seven of live mice will be of $i v^{-/-}$genotype, and 4/7 will be $\mathrm{Nodal}^{+/-}$, should this mutation suppress lethality in a subgroup of mice destined to die. Moreover, the ratio of $i v^{-1-}$ and $\mathrm{Nodal}^{+/-}$animals will now be not a simple product of their individual ratios, rather this genotype will be enriched, and is predicted to occur at a $2 / 7$ rate: $1 / 7$ stems from WW:CC (or CC:WW) segregants and 1/7 from rescued WC:WC segregants (Figure 3B). The observed result of these crosses is summarized in Table 3. Amongst 202 offspring, we found $66 \mathrm{iv}^{-/-}$and $103 \mathrm{Nodal}^{+/-}$ animals. Thirty-three mice were both $i v^{-/-}$and $\mathrm{Nodal}^{+/-}$. These numbers do not support the Nodal gene being the hypothetical lra1 gene. Rather, they show that $l r d$ mutation causes $50 \%$ lethality in Nodal heterozygotes as well.

\section{DISCUSSION}

We propose DNA's chirality and its asymmetric mode of replication as a potential source for installing binary imprints on the

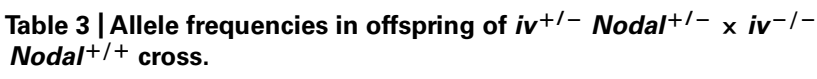

\begin{tabular}{llll}
\hline $\boldsymbol{n = 2 0 2}$ & Conv. expected & SSIS expected & Observed \\
\hline$I V^{-/-}$ & $1 / 3=67.3$ & $3 / 7=86.6$ & 66 \\
Nodal & $+/-$ & $4 / 7=115.4$ & 103 \\
$\mathrm{iV}^{-/-}$and Nodal ${ }^{+/-}$ & $1 / 2=101$ & $2 / 7=57.7$ & 32
\end{tabular}

chromatin fiber, and selective segregation of thus differentiated sister chromatids to sister cells as a novel and largely uncharacterized molecular mechanism associated with asymmetric cell divisions. The $l r d$-dependent segregation bias of mouse chromosome 7 sister chromatids in mitotic recombination experiments involving ES cells, endoderm cells, and neuroectoderm (Armakolas and Klar, 2007) cells could represent a case for selective sister chromatid segregation. Even though direct evidence for this interpretation is still missing, it led us to further investigate the phenotype of the lrd mouse mutant. In our model lrd functions to "sort" and selectively segregate sister chromatids based on their replication history in a WW:CC fashion. This L/R symmetry-breaking asymmetric cell division would be oriented along the $\mathrm{L} / \mathrm{R}$ axis, positional information for it would presumably come from polarized cytoskeleton (Vandenberg and Levin, 2009). Randomization of chromatid segregation in $\mathrm{iv}^{-1-}$ mice would lead to $25 \%$ normal organ situs (WW:CC segregants), 25\% situs inversus (CC:WW segregants), and 50\% death (WC:WC segregants). The 50 situs solitus : 50 situs inversus distribution in lrd mutant live animals has been described in numerous studies, therefore we only focused on assessment of lethality ratios by studying Mendelian inheritance of $l r d$ mutant alleles in appropriate genetic crosses. In order to eliminate potential allele-specific or genetic backgroundspecific artifacts, we analyzed two distinct lrd null alleles that had been outbred onto mixed backgrounds. Both crosses revealed $1 \mathrm{rd}$ homozygous mutant animals at rates $50 \%$ below Mendelian predictions. This result is consistent with our SSIS hypothesis even though it does not provide definitive proof of it. Nearly all studies of mouse laterality stress only the $50 \%$ situs solitus: $50 \%$ situs inversus phenotype of $i v^{-1-}$ mice and ignore the $50 \%$ lethality phenotype. Approximately 50\% lethality was first noted by Layton (1978) in one of the earliest studies of $i v$ mutant crosses. Our results presented here with iv confirmed the estimations of Layton (1978) and extended it to the newly made Lrd-Neo-GFP allele. One caveat for our SSIS explanation is that the original iv allele might be a leaky missense mutation generating the observed effects. It was therefore important to investigate phenotypes of a different allele, which is why we used the second Lrd-Neo-GFP allele for our analysis. A third allele was investigated previously, but the analysis was very limited to draw conclusions regarding lethality (Supp et al., 1999). Unfortunately, that allele was not saved (M. Bruckner, personal communication).

We next sought to test another prediction of our model, namely that heterozygosity for the hypothetical lral gene would rescue WC:WC segregants. The rationale therefore is that strand-specific imprinting of lral would lead to conflicting (ON/OFF) lral epialleles in both WC:WC sister cells. If one allele of lra1 is a null allele (due to heterozygosity), then different epialleles cannot be conflicting anymore (Figure 3A). We chose a reverse genetics approach and tested the Nodal gene as a possible candidate for lra1. Analysis of $>200$ offspring did not show a protective function for Nodal heterozygosity in lrd mutant animals: therefore, Nodal cannot be lral. We did however confirm the 50\% lethality phenotype of $i^{-/-}$genotype, indicating that lethality was not affected by Nodal gene dosage.

We have eliminated Nodal as a candidate for lral, and its ActR2B receptor can also be disregarded, because a study from 
En Li's laboratory (Oh and Li, 2002) recorded situs ambiguous (pulmonary isomerism) in around $40 \%$ of $i v^{-/-}$ActR2B $B^{+/-}$ embryos. If $A c t R 2 B$ were lral, then the SSIS model predicts occurrence of situs solitus and situs inversus only. The Nodal signaling pathway is highly complex and regulated by numerous factors on several levels (Schier, 2009). After Nodal precursor is activated by proprotein convertases and released into extracellular space, Lefty proteins limit its activity via a reaction-diffusion mechanism. Nodal binding to activin receptors is assisted by distinct co-receptors, and sometimes Nodal binds in conjunction with other TGF- $\beta$ molecules as a heterodimer. Moreover, evidence from zebrafish suggests tight post-transcriptional control of Nodal, Lefty and activin receptor gene expression by microRNAs (Schier, 2009). Given this level of complexity, a reverse genetic screen is unfeasible to tackle the identity of lral gene in the first step of L/R asymmetry development.

Interestingly, two recent studies have suggested that the nematode C. elegans employs SSIS mechanism during Neuronal asymmetry development. A study from Michael Levin's research group provides genetic support that an SSIS-type asymmetric cell division operates in olfactory neuron development, although the evidence has not been interpreted as such by the authors. The Levin laboratory has a long-standing interest in vertebrate $\mathrm{L} / \mathrm{R}$ axis development, and has highlighted the role of the cytoskeleton in cellular polarization for years (Aw and Levin, 2009; Vandenberg and Levin, 2009, 2010). It had come to the authors' attention that Arabidopsis mutants affecting radial flower symmetry were mapped to alphatubulin and a gamma-tubulin associated protein (Lobikin et al., 2012). Remarkably, introducing the same alpha-tubulin mutation into Xenopus 1-cell embryos resulted in development of heterotaxia, and in cultured human HL-60 cells it disturbed the leftward bias (with respect to the nucleus-centrosome axis) of pseudopodia protrusion. In addition, C. elegans "AWC" olfactory neural asymmetry was also affected by mutating a tubulin homolog (TBA-9, $75 \%$ amino acid identity with Arabidopsis alpha-tubulin) at two conserved amino acids. In wt worms, the AWC neuron is in the "ON" state $\left(\mathrm{AWC}^{\mathrm{ON}}\right)$ on one body side and in the "OFF" state $\left(\mathrm{AWC}{ }^{\mathrm{OFF}}\right)$ on the other body side; sidedness is stochastic (Chang etal., 2011). This developmental asymmetry can be visualized by introducing the "str-2p::GFP" fluorescent GFP construct in the genome. Importantly, chromosomal integration site of the GFP transgene is irrelevant for faithful $\mathrm{AWC}^{\mathrm{ON}}$ versus $\mathrm{AWC}^{\mathrm{OFF}}$ discrimination, indicating that the cause for asymmetric GFP expression acts in trans for the transgene. The authors chose this model system for body asymmetry development studies, because the $\mathrm{AWC}^{\mathrm{ON}}$ and $\mathrm{AWC} \mathrm{OFF}^{\mathrm{OF}}$ cells show cytoskeletal polarization and asymmetric calcium signaling, which is sensitive to the microtubule depolymerizing drug nocodazole (Chang et al., 2011). Overexpression of wt TBA-9 tubulin in transgenic worms causes only mild laterality defects, with $82 \%$ of worms displaying the normal $1 \mathrm{AWC} \mathrm{ON}^{\mathrm{N}} / 1 \mathrm{AWC} \mathrm{OFF}^{\mathrm{FF}}$ phenotype. Overexpression of mutant TBA- 9 , in contrast, results in $42 \%$ normal $1 \mathrm{AWC}^{\mathrm{ON}} / 1 \mathrm{AWC}^{\mathrm{OFF}}$ and $45 \%$ novel $2 \mathrm{AWC}^{\mathrm{ON}}$ "heterotaxic" phenotype. This roughly 50:50 distribution is consistent with a SSIS mechanism operating in the mother AWC cell (Figure 4). We hypothesize that one daughter inherits normally two $\mathrm{AWC}^{\mathrm{ON}}$ epialleles and the other two $\mathrm{AWC}^{\mathrm{OFF}}$ epialleles are inherited by the other daughter cell (WW:CC segregation). Unlike our SSIS model for mouse $\mathrm{L} / \mathrm{R}$ axis development, this asymmetric distribution of sister chromatids in the worm occurs irrespective of the L/R body axis. We propose that introduction of mutated tubulin renders the AWC cell's cytoskeleton unable to direct selective chromatid segregation in mitosis, hence a novel WC:WC segregation results at $50 \%$ frequency. A simple explanation for $2 \mathrm{AWC}^{\mathrm{ON}}$ phenotype in WC:WC segregants would be dominance of the $\mathrm{AWC}^{\mathrm{ON}}$ over the $\mathrm{AWC}^{\mathrm{OFF}}$ epiallele.

A second study implicating an SSIS-like asymmetric cell division in C. elegans neuronal asymmetry development has been recently published by Horvitz/Stillman laboratories (Nakano et al., 2011). Here, a GFP-reporter screen served to isolate mutants that changed the paired asymmetric MI motor neuron/e3D epidermal cell pair to a symmetrical e3D cell pair on both sides of the brain. Positional cloning identified a gain-of-function mutation in a histone $\mathrm{H} 3$ gene that deleted its last 11 amino acids, thereby impairing the ability to form $\mathrm{H} 3 / \mathrm{H} 4$ tetramers during chromatin assembly. Likewise, RNAi against chromatin assembly factor 1 (CAF-1) or PCNA pheno-copied the $\mathrm{H} 3$ mutant. The authors suggest that newly lagging strand synthesized DNA contains elevated levels of PCNA and associated CAF-1 containing histone chaperone complex, which deposits higher nucleosome density. This could represent an epigenetic imprint in itself, or serve to nucleate covalent chromatin modifications. The latter seems somewhat more likely, since the epigenetic imprint is transmitted through several mitoses, as it is the MI/e3D great-great-grandmother cell that directs development of distinct cell fates three cell divisions later on. Like in our SSIS model, selective segregation of epigenetically differentiated sister chromatids is an integral part of the authors' model. However, neither direct nor indirect evidence is presented. Because genetic evidence suggests that mutated tubulin (Lobikin et al., 2012) randomizes (the normally selective) chromatid segregation during an $\mathrm{AWC}^{\mathrm{ON}} / \mathrm{AWC}^{\mathrm{OFF}}$ olfactory neuron asymmetry generating cell division, we propose to test whether mutated TBA-9 also affects the MI/e3D neuronal asymmetry.

The SSIS model is conceptually based on three aspects: (i) differential chromatin imprinting during inherently lagging versus leading strand replication, (ii) one or several genes who's expression is affected by this imprint, and (iii) a segregator that identifies and "sorts" epigenetically differentiated sister chromatids by operating at sister centromeres in mitosis. We have presented genetic evidence for (iii), namely that $l r d$ acts as a segregator in a $\mathrm{L} / \mathrm{R}$ axis defining cell division in mouse. In contrast, Nakano et al. (2011) have provided evidence for (i), but have not identified the segregator. If the segregator can be identified, C. elegans will be excellently suited to use forward genetics to identify the gene or set of genes (ii) that are imprinted and selectively segregated, as outlined in our iv ${ }^{-/-}$, Nodal ${ }^{+/-}$breeding experiment.

We have highlighted two studies that support a SSIS-type mechanism in the development of neuronal asymmetries in C. elegans. Based on genetics of psychosis development in human carriers of balanced chromosome 11 translocations, we have previously proposed that a similar mechanism may operate during human brain lateralization (Klar, 2004). Analogous to our model for body laterality development, brain laterality development would also initiate with a single critical asymmetric cell division, where chromosome 


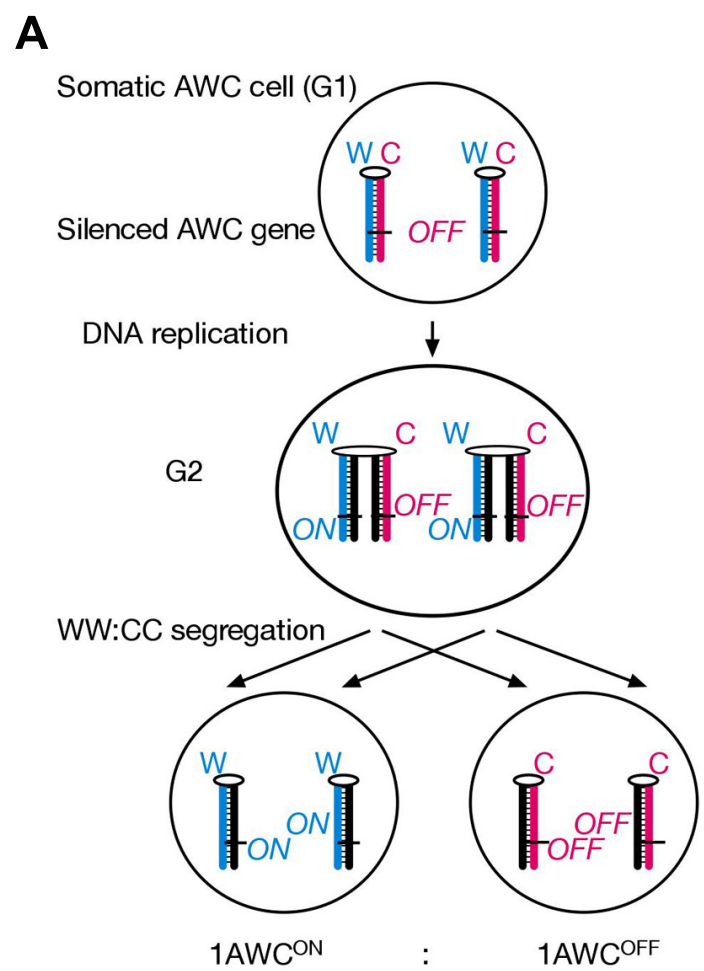

Sister cells with different fates
FIGURE 4 | A finding published by Dr. Michael Levin's laboratory is interpreted to suggest that an SSIS-like mechanism operates during olfactory neuron asymmetry development in $\boldsymbol{C}$. elegans. (A) An AWC precursor cell undergoes asymmetric cell division and selectively segregates epigenetically differentiated sister chromatids containing an AWC

master-regulator gene in a WW: CC fashion, such that always a
B

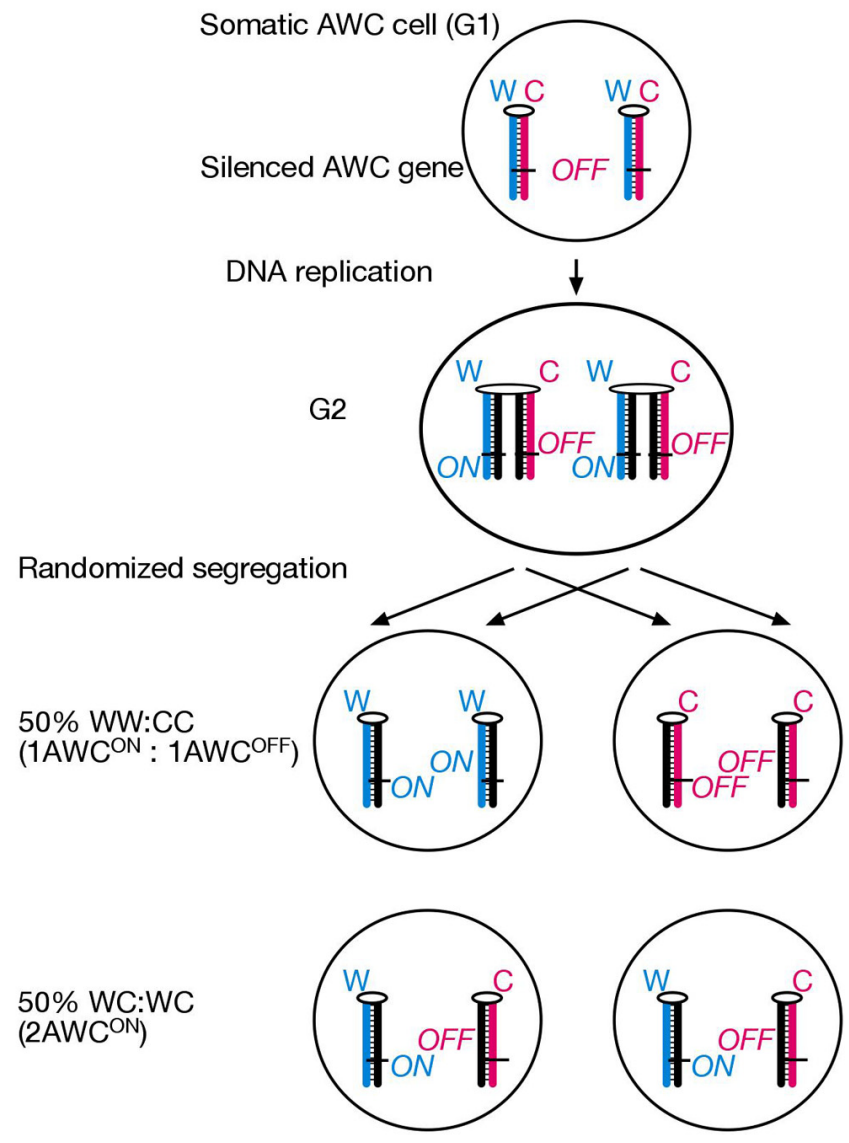

Sister cells

1 AWCON/1AWCOFF olfactory cell pair develops in each worm. (B) Embryos transgenic for mutated (but not wild-type) tubulin developed either $1 \mathrm{AWC} \mathrm{ON}_{/ 1} \mathrm{AWC} \mathrm{OFF}^{\mathrm{O}}$ or $2 \mathrm{AWC} \mathrm{ON}^{\mathrm{N}}$ olfactory neuron cells at a roughly 50-50 frequency. We explain this result by the SSIS model due to randomized chromatid segregation during the critical $\mathrm{AWC}^{\mathrm{ON}} / \mathrm{AWC}^{\mathrm{OFF}}$ neuron generating cell division due to the tubulin mutation.
11 sister chromatids are selectively segregated in a WW:CC fashion. If one chromosome 11 homolog is fused to the centromere of another chromosome not undergoing selective mitotic segregation, then WW:CC and WC:WC segregation for chromsome 11 are expected to occur at equal frequencies. Fifty per cent incidence of psychosis development in four different families with balanced chromosome 11 translocations support our hypothesis (Singh and Klar, 2007).

Whether asymmetric cell divisions elsewhere during normal tissue homeostasis employ a SSIS mechanism remains to be determined. If they do exist, then somatic chromosomal translocations could potentially randomize these asymmetric cell divisions and initiate tumorigenesis. An example would be a resting tissue stem cell that only enters the cell cycle upon tissue injury. It asymmetrically divides to produce a rapidly-proliferating transiently amplifying stem cell. This cellular asymmetry development would be controlled by asymmetric segregation of cytoplasmic determinants, but also by WW:CC segregation of epigenetically differentiated sister chromatids, where cell cycle promoting genes remain silenced in the mother cell, but poised for expression in the transiently amplifying daughter cell. A chromosomal translocation involving the chromosome undergoing selective segregation in the tissue stem cell could therefore change the WW:CC pattern to a WC:WC pattern. As a result, the resting tissue stem cell would acquire proliferative capacities of the transiently amplifying stem cell, leading to neoplasia. Additional oncogenic mutations will eventually render this cell growth cancerous. Despite this example being rather simplistic, it should be appreciated that genes controlling asymmetric cell division are increasingly recognized as tumor suppressors. Drosophila brat and prospero mutants, for example, fail to undergo asymmetric neuroblast cell divisions, and develop larval brain tumors (Betschinger et al., 2006). We suggest that somatic chromosomal translocations in tissue stem cells could affect biased segregation of sister chromatids, 
and change strictly asymmetrically dividing stem cells to stem cells that undergo symmetrical cell divisions in terms of epigenetic imprints on differentiated sister chromatids distal to the translocation breakpoint.

Curiously, a 1992 study published in The Lancet (Sandson et al., 1992) found a correlation of abberrant brain laterality development and breast cancer. Right-handed breast cancer patients and healthy controls were subjected to computer tomographic brain scans. Eighty-two per cent of control subjects showed left hemispheric dominance, whereas in the breast cancer group this number was reduced to $51 \%$. Although this study should be cautiously interpreted until replicated elsewhere, it certainly suggests that brain laterality- and breast cancer-development share a common genetic pathway (Klar, 2011). We suggest that this pathway controls asymmetric cell divisions during embryonic brain development, and during cell turnover in the lactiferous duct upon periodic hormonal growth stimulation. Hence, improving our understanding of vertebrate laterality development could eventually impact on cancer prevention and treatment.

Taken together, 50\% lethality phenotype in the lrd mouse mutant supports predictions made by the SSIS model for laterality development. Here, $l r d$ is part of a cellular mechanism that selectively segregates epigenetically differentiated sister chromatids concerning their replication history with respect to a cytoskeletonbased early L/R axis (Klar, 2008; Vandenberg and Levin, 2010; Lobikin et al., 2012). The overwhelming majority of studies on lrd in the mouse have focused on its role in conferring nodal cilia motility. This is understandable, since genetics of spontaneous and targeted mouse mutations affecting laterality development have generally pointed to a central role for motile nodal cilia. Moreover, the earliest known molecular L/R asymmetries appear after node formation in the mouse. In chicken and Xenopus, in contrast, earlier asymmetries involving Gap-junctional communication (Levin and Mercola, 1999), $\mathrm{H}^{+} / \mathrm{K}^{+}$ATPase activity (Levin et al., 2002), and serotonin signaling (Fukumoto et al., 2005) have been identified. As many of the studies on earlier asymmetry determinants in frogs and chicken involved embryo-exposure to pharmacological inhibitors, mouse embryo-culture protocols will need to vastly improve until replication can even be considered. In this regard it is noteworthy that one of the leading laboratories for mouse embryo in vitro culture has recently tested the relationship of nodal cilia emitted force and asymmetry development in

\section{REFERENCES}

Armakolas, A., and Klar, A. J. (2006). Cell type regulates selective segregation of mouse chromosome 7 DNA strands in mitosis. Science 311, 11461149.

Armakolas, A., and Klar, A. J. (2007). Left-right dynein motor implicated in selective chromatid segregation in mouse cells. Science 315, 100-101.

Aw, S., and Levin, M. (2009). Is left-right asymmetry a form of planar cell polarity? Development 136, 355-366.

Betschinger, J., Mechtler, K., and Knoblich, J. A. (2006). Asymmetric segregation of the tumor

several mouse mutants affecting cilia biogenesis and motility (Shinohara et al., 2012). Surprisingly, the authors found that as few as two motile nodal cilia were sufficient to break bilateral symmetry. These data are rather compatible with the "2-cilia hypothesis," which was initially postulated by the Hirokawa, Brueckner, and Tabin laboratories (Okada et al., 1999; McGrath et al., 2003; Tabin and Vogan, 2003). Here, mechanical force exerted by beating nodal cilia is read out by mechanosensory cilia, which are associated with polycystin-2 (a calcium release channel) to induce left-sided calcium signaling. Hence loss of polycystin-2 is predicted to ablate calcium and Nodal signaling altogether. Pennekamp et al. (2002) indeed found loss of Nodal expression in the majority of polycystin2 deficient embryos, however the Nodal downstream target Pitx2 showed bilateral expression. Clearly how body laterality is initially developed, whether in visceral organs or in the nervous system, remains controversial thus far. Further work is needed to differentiate if any of the prevailing hypotheses can satisfactorily explain body laterality development. The SSIS model is simple to understand in that an asymmetric cell division constitutes the root cause of development. In this model, developmental decisions are made through particulate matter consisting of ON/OFF epigenetic states of gene expression of developmentally important gene(s). Thus, in addition to acting as genetic material, DNA strands can provide the basis for evolution, cancer and development (Furusawa, 2011).

\section{ACKNOWLEDGMENTS}

We are grateful to Dr. Martina Brueckner (Yale University, New Haven, CT) for sharing the Lrd-Neo-GFP allele and to Dr. Michael Kuehn (Frederick National Laboratory, MD) for sharing the delta Nodal allele. We would like to thank Dr. Mark Lewandoski for discussions and use of his laboratory facilities and Lisa Dodge for mouse husbandry. The Intramural Research Program of the National Institutes of Health, Frederick National Laboratory for Cancer Research supports our research. Stephan Sauer is recipient of a long-term Fellowship from the Human Frontier Science Program Organization (LT-000444/2009).

\section{AUTHOR CONTRIBUTIONS}

Amar J. S. Klar and Stephan Sauer designed experiments, Stephan Sauer carried out experiments and collected data, Stephan Sauer and Amar J. S. Klar interpreted the data and wrote the manuscript.

of the left-right axis in chick and frog embryos. Curr. Biol. 15 , 794-803.

Furusawa, M. (2011). Implications of double-stranded DNA structure for development, cancer and evolution. Open J. Genet. 1, 78-87.

Gardner, R. L. (2010). Normal bias in the direction of fetal rotation depends on blastomere composition during early cleavage in the mouse. PLoS ONE 5, e9610. doi: 10.1371/journal.pone.0009610

Hummel, K. P., and Chapman, D. B. (1959). Visceral inversion and associated anomalies in the mouse. J. Hered. 50, 9-13.
Klar, A. J. (1994). A model for specification of the left-right axis in vertebrates. Trends Genet. 10, 392-396.

Klar, A. J. (2004). An epigenetic hypothesis for human brain laterality, handedness, and psychosis development. Cold Spring Harb. Symp. Quant. Biol. 69, 499-506.

Klar, A. J. (2011). Breast cancer predisposition and brain hemispheric laterality specification likely share a common genetic cause. Breast Dis. $33,49-52$.

Klar, A. J. (2007). Lessons learned from studies of fission yeast mating-type switching and silencing. Annu. Rev. Genet. 41, 213-236. 
Klar, A. J. (2008). Support for the selective chromatid segregation hypothesis advanced for the mechanism of left-right body axis development in mice. Breast Dis. 29, 47-56.

Layton, W. M. Jr. (1978). Heart malformations in mice homozygous for a gene causing situs inversus. Birth Defects Orig. Artic. Ser. 14, 277-293.

Levin, M., and Mercola, M. (1999). Gap junction-mediated transfer of leftright patterning signals in the early chick blastoderm is upstream of Shh asymmetry in the node. Development 126, 4703-4714.

Levin, M., Thorlin, T., Robinson, K. R., Nogi, T., and Mercola, M. (2002). Asymmetries in $\mathrm{H}^{+} / \mathrm{K}^{+}$-ATPase and cell membrane potentials comprise a very early step in leftright patterning. Cell 111, 77-89.

Liu, P., Jenkins, N. A., and Copeland, N. G. (2002). Efficient Cre-loxPinduced mitotic recombination in mouse embryonic stem cells. Nat. Genet. 30, 66-72.

Lobikin, M., Wang, G., Xu, J., Hsieh, Y. W., Chuang, C. F., Lemire, J. M., et al. (2012). Early, nonciliary role for microtubule proteins in left-right patterning is conserved across kingdoms. Proc. Natl. Acad. Sci. U.S.A. 109, 12586-12591.

Lowe, L. A., Yamada, S., and Kuehn, M. R. (2001). Genetic dissection of nodal function in patterning the mouse embryo. Development 128, 1831-1843.

McGrath, J., Somlo, S., Makova, S., Tian, X., and Brueckner, M. (2003). Two populations of node monocilia initiate left-right asymmetry in the mouse. Cell 114, 61-73.

Muller, P., Rogers, K. W., Jordan, B. M., Lee, J. S., Robson, D., Ramanathan, S., et al. (2012). Differential diffusivity of Nodal and Lefty underlies a reactiondiffusion patterning system. Science 336, 721-724.

Nakamura, T., and Hamada, H. (2012). Left-right patterning: conserved and divergent mechanisms. Development 139, 3257-3262.

Nakamura, T., Mine, N., Nakaguchi, E., Mochizuki, A., Yamamoto, M., Yashiro, K., etal. (2006). Generation of robust left-right asymmetry in the mouse embryo requires a self-enhancement and lateralinhibition system. Dev. Cell 11, 495-504.

Nakano, S., Stillman, B., and Horvitz, H R. (2011). Replication-coupled chromatin assembly generates a neuronal bilateral asymmetry in C. elegans. Cell 147, 1525-1536.

Nonaka, S., Tanaka, Y., Okada, Y., Takeda, S., Harada, A., Kanai, Y. et al. (1998). Randomization of leftright asymmetry due to loss of nodal cilia generating leftward flow of extraembryonic fluid in mice lacking KIF3B motor protein. Cell 95, 829-837.

Nonaka, S., Shiratori, H., Saijoh, Y., and Hamada, H. (2002). Determination of left-right patterning of the mouse embryo by artificial nodal flow. Nature 418, 96-99.

Oh, S. P., and Li, E. (2002) Gene-dosage-sensitive genetic interactions between inversus viscerum (iv), nodal, and activin type IIB receptor (ActRIIB) genes in asymmetrical patterning of the visceral organs along the left-right axis. Dev. Dyn. 224, 279-290.

Okada, Y., Nonaka, S., Tanaka, Y., Saijoh, Y., Hamada, H., and Hirokawa, N. (1999). Abnormal nodal flow precedes situs inversus in iv and inv mice. Mol. Cell 4, 459-468.

Pennekamp, P., Karcher, C., Fischer, A. Schweickert, A., Skryabin, B., Horst,
J., etal. (2002). The ion channel polycystin-2 is required for left-right axis determination in mice. Curr. Biol. 12, 938-943.

Sandson, T. A., Wen, P. Y., and LeMay, M. (1992). Reversed cerebral asymmetry in women with breast cancer. Lancet 339, 523-524.

Schier, A. F. (2009). Nodal morphogens. Cold Spring Harb. Perspect. Biol. 1, a003459.

Shinohara, K., Kawasumi, A., Takamatsu, A., Yoshiba, S., Botilde, Y. Motoyama, N., etal. (2012). Two rotating cilia in the node cavity are sufficient to break left-right symmetry in the mouse embryo. Nat. Commun. 3, 622.

Singh, G., and Klar, A. J. (2007). A hypothesis for how chromosome 11 translocations cause psychiatric disorders. Genetics 177, 1259-1262.

Supp, D. M., Brueckner, M., Kuehn, M. R., Witte, D. P., Lowe, L. A., McGrath, J., et al. (1999). Targeted deletion of the ATP binding domain of left-right dynein confirms its role in specifying development of leftright asymmetries. Development 126 , 5495-5504.

Supp, D. M., Witte, D. P., Potter, S. S. and Brueckner, M. (1997). Mutation of an axonemal dynein affects leftright asymmetry in inversus viscerum mice. Nature 389, 963-966.

Tabin, C. (2005). Do we know anything about how left-right asymmetry is first established in the vertebrate embryo? J. Mol. Histol. 36, 317-323.

Tabin, C. J., and Vogan, K. J. (2003). A two-cilia model for vertebrate leftright axis specification. Genes Dev. 17, $1-6$

Vandenberg, L. N., and Levin, M. (2010). Far from solved: a perspective on what we know about early mechanisms of left-right asymmetry. Dev. Dyn. 239, 3131-3146.

Vandenberg, L. N., and Levin, M. (2009). Perspectives and open problems in the early phases of left-right patterning. Semin. Cell Dev. Biol. 20, 456-463.

Yamamoto, M., Mine, N., Mochida, K., Sakai, Y., Saijoh, Y., Meno, C.,et al. (2003). Nodal signaling induces the midline barrier by activating Nodal expression in the lateral plate. Development 130, 1795-1804.

Conflict of Interest Statement: The authors declare that the research was conducted in the absence of any commercial or financial relationships that could be construed as a potential conflict of interest.

Received: 07 September 2012; paper pending published: 07 October 2012; accepted: 25 October 2012; published online: 16 November 2012.

Citation: Sauer S and Klar AJS (2012) Left-right symmetry breaking in mice by left-right dynein may occur via a biased chromatid segregation mechanism, without directly involving the Nodal gene. Front. Oncol. 2:166. doi: 10.3389/fonc. 2012.00166

This article was submitted to Frontiers in Cancer Genetics, a specialty of Frontiers in Oncology.

Copyright (C) 2012 Sauer and Klar. This is an open-access article distributed under the terms of the Creative Commons Attribution License, which permits use, distribution and reproduction in other forums, provided the original authors and source are credited and subject to any copyright notices concerning any thirdparty graphics etc. 